\title{
Numerical study of anomalous refraction in photonic crystals
}

\author{
Suxia Yang, Tao Xu, and Harry Ruda* \\ Center for Advanced Nanotechnology, University of Toronto, Toronto, Ontario M5S 3E3, Canada \\ Michael Cowan \\ Departments of Physics and Chemistry, University of Toronto, Toronto, Ontario M5S 3H6, Canada
}

(Received 3 May 2005; published 19 August 2005)

\begin{abstract}
We present a theoretical study of light propagation through photonic crystal $(\mathrm{PhC})$ structures using an effective medium approximation based on equifrequency surface calculations. The procedure is illustrated by calculating bending, splitting and focusing phenomena in a slab of two-dimensional $\mathrm{PhC}$ using a Fourier imaging technique. In the passband above the first stop band, wave propagation was found to depend dramatically on both light frequency and incident direction. This propagation anisotropy leads to very large negative refraction along the [11] crystal direction. Light emitted from a line source outside the PhC slab can be focused, divided into subbeams inside the $\mathrm{PhC}$, bent negatively, and refocused again on the other side the slab. The results agree well with the field calculated using a finite-difference time-domain method and can be straightforwardly applied to more complicated $\mathrm{PhC}$ structures, such as three-dimensional PhC structures. The imaging properties of a $\mathrm{PhC}$ slab are also discussed.
\end{abstract}

DOI: 10.1103/PhysRevB.72.075128 PACS number(s): 78.20.Ci, 42.70.Qs, 42.25.-p, 42.30.-d

\section{INTRODUCTION}

Photonic crystals ( $\mathrm{PhC}$ 's) have attracted tremendous attention since the concept was proposed in the late 1980s, ${ }^{1,2}$ due to the fact that they provide excellent model systems for fundamental research and owing to their potential applications in novel optical circuits and devices. Early experimental and theoretical study of PhC's has focused on infinite crystals and the existence of full photonic band gaps where light cannot propagate along any crystal direction. In recent years, anomalous light behaviors in PhC's, such as negative refraction and the superlens effect, have attracted much attention. ${ }^{3-15}$ Negative refraction is an intriguing phenomenon first found in left-handed materials (LHM's). The concept of LHM's is based on the proposal by Veselago about 40 years ago. ${ }^{16}$ In a LHM both the effective permeability $(\mu)$ and permittivity $(\varepsilon)$ are negative; thus, the effective refractive index is also negative. Study of light behavior in PhC's, however, proves that negative $\mu$ and $\varepsilon$ are not necessary for achieving negative refraction. It has recently been demonstrated that all-angle negative refraction (AANR) can be obtained for a photonic band having a positive group velocity and a positive refractive index in a $\mathrm{PhC} .{ }^{15}$ This AANR can be used to focus a point source through a photonic crystal slab and achieve subwavelength resolution from the enhancement of evanescent waves.

Although there have been quite a few papers presenting field simulation and showing focusing effects, there has not been a systematic study of the properties of $\mathrm{PhC}$ imaging. $3,4,6-8,10,11,15$ Notomi gave a brief discussion in his paper on imaging in $\mathrm{PhC}$ 's but he only focused on the refractionlike effect in the vicinity of the photonic band gap and did not consider multiple reflections which usually exist inside a real $\mathrm{PhC}$ slab because of the mismatch of the effective refractive index. ${ }^{14}$ On the other hand, most previous field simulations were calculated using a finite-difference time- domain (FDTD) method. The FDTD method is a relatively straightforward method based on discretizing Maxwell's equation in real space and time. However, this approach is not physically intuitive, and since it requires intensive computations, it is very time consuming to calculate realistically sized samples, especially for three-dimensional (3D) structures. Recently several other methods-namely, a multiplescattering method for a $\mathrm{PhC}$ slab of finite size, ${ }^{17}$ a layered Korringa-Kohn-Rostoker method for a PhC slab of laterally infinite extension, ${ }^{5}$ and a method based on Bloch modes to analyze $\mathrm{PhC}$ waveguides ${ }^{18}$ - have been developed. In this paper, we present a different method to calculate the propagation of light through $\mathrm{PhC}$ structures. By approximating the $\mathrm{PhC}$ as an effective medium based on its dispersion relations, we can use a Fourier imaging technique (FIT) to study imaging in $\mathrm{PhC}$ structures. We demonstrate this technique by investigating the imaging properties, negative refraction, collimation, and focusing in a slab of 2D PhC.

Light behavior in a $\mathrm{PhC}$ can be analyzed from its band structure and/or equifrequency surfaces (EFS's). The dispersion relation is calculated either directly in the frequency domain using methods such as the plane-wave expansion or indirectly in real space using a FDTD method followed by a Fourier transformation of a time-varying response. Here we calculate the dispersion relation using the MIT photonic band (MPB) package developed by Johnson and Joannopoulos. ${ }^{19}$ Using approximate boundary conditions we can then model the $\mathrm{PhC}$ as an effective medium whose properties are given by the calculated dispersion relation and equifrequency surface. A simple FIT is then used to simulate a line light source propagating through a slab of $2 \mathrm{D} \mathrm{PhC}$ by decomposing the source into plane waves. It should be noted that this FIT for light was adopted from a Fourier imaging method that we recently developed to study the focusing effect of sound in a $3 \mathrm{D}$ phononic crystal. ${ }^{20}$ But here we improve this method by including all the multiple reflections inside the $\mathrm{PhC}$ slab. Since most pervious work is focused either on the lowest 

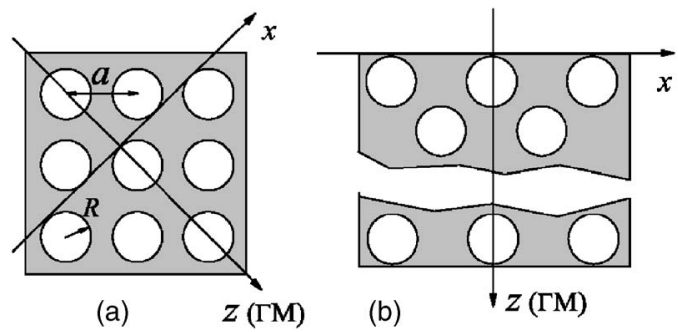

FIG. 1. Left: square-lattice crystal structure, where $a$ is the lattice constant and $R$ is the radius. Right: cross section of a slab of a 2D $\mathrm{PhC}$ with this crystal structure, where the surface normal is along the $\Gamma M$ direction and the air holes are aligned along the $y$ direction which is normal to the cross section.

band or around the band edges, we choose to study the phenomena around frequencies at the middle of a second band. However, one should note that our method is not limited to this frequency range or structure, and the goal of this paper is to illustrate the effective medium and Fourier imaging techniques, rather than searching for the best frequency regime for imaging.

\section{MODELING}

Without losing generality, we choose to study a $2 \mathrm{D} \mathrm{PhC}$ consisting of a square lattice (having lattice constant $a$ ) of air holes with a radius of $0.35 a$ in a dielectric medium $(\varepsilon=12)$ as shown in Fig. 1, where the surface normal is along the $\Gamma M$ direction ([11] direction) and the air holes are aligned along the $y$ direction which is normal to the cross section. This is the same PhC structure as studied by Yu and Fan ${ }^{21}$ and Luo et al. ${ }^{15}$ respectively, in two recent papers. In this paper, we focus on the modes with the magnetic field along the axis of the air holes. These modes are sometimes called transverseelectric (TE) modes, since the electric field is normal to the axis of the air holes. One should note that this notation is different from the traditional definition, in which polarization is defined according to the relative orientation of the field respect to the incident plane. We are interested in the TE modes at the dimensionless frequency $\Omega=0.3$ in the second band. Here the dimensionless frequency $\Omega$ is defined as $\Omega$ $=\omega a /(2 \pi c)$, where $\omega$ is the angular frequency, $a$ the lattice constant, and $c$ the speed of light in vacuum. The EFS's at different frequencies in this frequency range are plotted in the first Brillouin zone in Fig. 2. It can be seen that the shape of the EFS depends strongly on the frequency. At the frequency $\Omega=0.26$, there are gaps along the $\Gamma M$ direction. As the frequency becomes higher, the gaps disappear and the EFS shrinks toward the $\Gamma$ point. The EFS has a square shape at frequencies around $\Omega=0.28$ but becomes more circular at $\Omega=0.32$. The energy flow direction is determined by the group velocity $v_{g}$ which is defined by $\nabla_{k} \omega$. In other words, $v_{g}$ is always normal to the EFS and pointing toward the EFS with a higher frequency. Since the EFS shrinks toward the center as the frequency gets higher, we can expect that light will propagate inward in this frequency range. For $\Omega \simeq 0.28$, light will be collimated inside this $\mathrm{PhC}$ due to the square shape of the EFS which determines that the group velocities

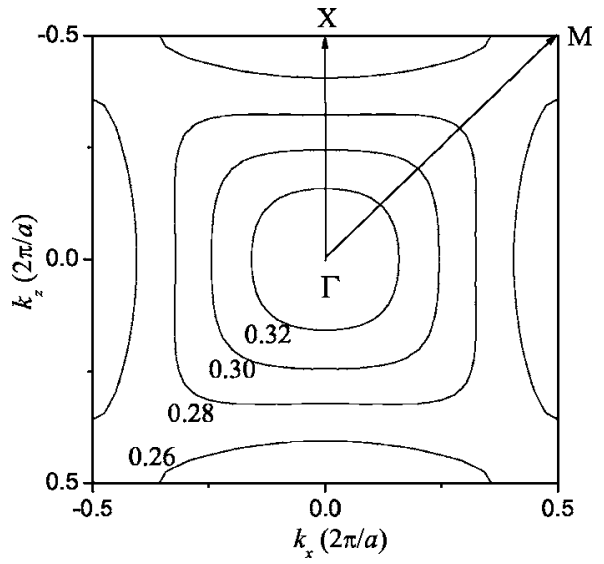

FIG. 2. Equifrequency surfaces in the first Brillouin zone around the frequency 0.3 .

of waves with different incident directions will have the same direction inside the $\mathrm{PhC}$.

Whether a phase velocity is meaningful in an infinite $\mathrm{PhC}$ remains an open question; however, we have shown, both theoretically and experimentally for phononic crystals, that an effective phase velocity is indeed an important parameter when dealing with boundary conditions of ultrasonic waves traveling through a 3D phononic crystal slab. ${ }^{22,23}$ The phase velocity can be determined from the cumulative phase relative to the input field, and it can only be understood when we study the EFS in an extended or a periodic Brillouin zone (BZ) scheme. To illustrate this idea in detail, we plot in Fig. 3 the EFS's at $\Omega=0.30$ in a periodic BZ scheme where the coordinate system has been rotated by $45^{\circ}$ so that $k_{x}^{\prime}$ is aligned along the $x$ direction and $k_{z}^{\prime}$ along the $z$ direction as shown in Fig. 1(b). Note that we have replaced the top EFS in Fig. 3 with the EFS in air in order to study light traveling from air into the PhC. Since the EFS outside of the region between the two dot-dashed lines cannot couple to light in air due to a mismatch of the wave vectors, we are only concerned the first BZ and the EFS translated by $k+(p-1) G_{\Gamma M}$,

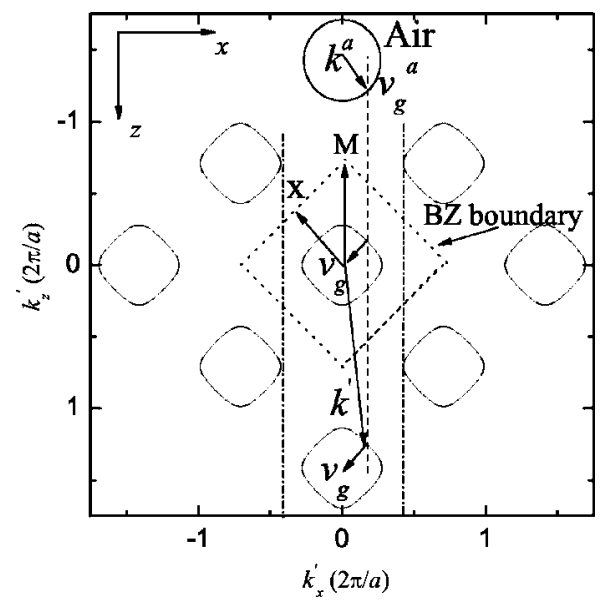

FIG. 3. Equifrequency surfaces of the $\mathrm{PhC}$ at $\Omega=0.30$ in a periodic Brillouin zone scheme. The top one is replaced with the equifrequency surface in air. The dotted square defines the first BZ. The dashed line helps to show that $k_{x}$ is conserved. 


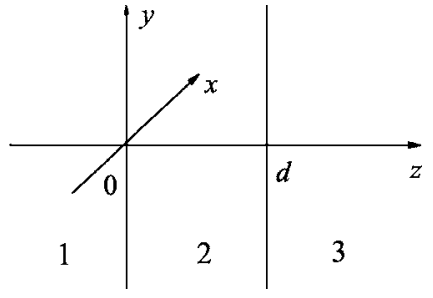

FIG. 4. Cross section of a dielectric slab (2) with thickness $d$ sandwiched between two semi-infinite media (1) and (2).

where $p$ is the number of pass bands and $G_{\Gamma M}$ is the shortest reciprocal vector along the $\Gamma M$ direction. The modes that we study here are in the second band; thus, $p=2$ and the translation equation is $k+G_{\Gamma M}$. We will call the corresponding $\mathrm{EFS} \mathrm{EFS}_{2}$ and the one in the first BZ EFS ${ }_{1}$ for simplicity. It is easy to see that for both $\mathrm{EFS}_{1}$ and $\mathrm{EFS}_{2}$, the group velocity is pointing toward the "negative side" with respect to the incident direction in air defined by $v_{g}^{a}$. This results in socalled negative refraction in a PhC. Both $\mathrm{EFS}_{1}$ and $\mathrm{EFS}_{2}$ give the same group velocity and thus will give the same propagation in an infinite crystal. However, when we deal with boundary conditions in finite photonic crystal structures, we need to use $k^{\prime}$ which corresponds to $\mathrm{EFS}_{2}$ as shown in Fig. 3 .

Here we derive a FIT through a single homogeneous and isotropic layer sandwiched between two semi-infinite media as shown in Fig. 4, where the surface normal is along the $z$ direction. We further assume that the plane of incidence is the $x z$ plane and the magnetic field is along the $y$ direction. We only consider this polarization $H=H_{y}$ because it is the mode that we will discuss in a $\mathrm{PhC}$ slab later in this paper. The magnetic field of a general plane-wave solution of the wave equation can be of the form

$$
H(z) e^{i(\omega t-\beta x)} .
$$

Here the parameter $\beta$ is the $x$ component of the propagating wave vector and it is a constant in all layers due to the boundary conditions. If we assume that the light source is in medium 1 and that wave propagates through medium 2 into medium 3, the magnetic field vector $H(z)$ can be written as

$$
\begin{aligned}
& H(z)=H_{y}(z)=A e^{-i k_{1} z}+B e^{i k_{1} z} \quad(z<0), \\
& =C e^{-i k_{2 z} z}+D e^{i k_{2 z} z} \quad(0<z<d), \\
& =F e^{-i k_{3 z}(z-d)} \quad(d<z) .
\end{aligned}
$$

Here the complex amplitudes $A, B, C, D$, and $F$ are constants and $k_{1 z}, k_{2 z}$, and $k_{3 z}$ are the $z$ components of the wave vectors in media 1,2 , and 3 , respectively. Using the boundary conditions that the tangential components of the electric and magnetic field vectors be conserved, we can obtain the parameters $B$ (reflected wave), $C$ (right-propagating wave inside medium 2), $D$ (left-propagating wave inside medium 2), and $F$ (transmitted wave) in terms of $A$ (incident wave):

$$
\begin{gathered}
F=A \frac{}{\left(k_{1 z}+k_{2 z} \varepsilon_{1} / \varepsilon_{2}\right)\left(k_{2 z}+k\right.}+ \\
C=\frac{1}{2}\left[1+\frac{k_{1 z}}{k_{2 z} \epsilon_{1} / \epsilon_{2}}\right] e^{i k_{2 z} d} F, \\
D=\frac{1}{2}\left[1-\frac{k_{1 z}}{k_{2 z} \epsilon_{1} / \epsilon_{2}}\right] e^{-i k_{2 z} d} F, \\
B=\frac{1}{2}\left[C+D-\frac{k_{2 z} \epsilon_{1} / \epsilon_{2}}{k_{1 z}}(C-D)\right] .
\end{gathered}
$$

Substituting the calculated values for $B, C, D$, and $F$ into Eqs. (2), provides a complete solution for the field anywhere in space for this particular plane wave.

A real light source can always be expanded into plane waves with different wave vectors by using Fourier analysis. Each plane wave $A^{m} e^{i k_{x}^{m} x}$ can then be treated individually using the above equations. The key point of the FIT is to calculate the phase and amplitude change of each plane wave in the image plane, using the equations above. Then, by taking the inverse Fourier transformation, we can simulate the field for image planes both inside and outside of this dielectric slab. For a uniform dielectric material, at a particular

frequency $\omega, k_{z}$ is related to $k_{x}$ by the equation $k_{z}=\sqrt{k^{2}-k_{x}^{2}}$, where $k=n \omega / c$ and $n$ is the refractive index of this material. Unlike a uniform dielectric material, $k_{z}$ cannot usually be directly related to $k_{x}$ using a simple equation in a PhC. However, we can calculate the EFS which gives $k_{z}$ as a function of $k_{x}$. In other words, we treat the $\mathrm{PhC}$ as an "effective medium" whose properties are characterized by its EFS.

\section{RESULTS AND DISCUSSION}

In this section, we first show some field simulations through an air/ $\mathrm{PhC}$ interface-i.e., where both air and the $\mathrm{PhC}$ are semi-infinite media. The surface normal of the $\mathrm{PhC}$ is along the $\Gamma M$ direction as shown in Fig. 1. Figure 5 gives the field patterns (magnitude of $H$ ) in the $x z$ plane for light emitted from a line source traveling through the air/PhC interface, where Figs. 5(a) and 5(c) (on the left) are calculated using the FIT and Figs. 5(b) and 5(d) (on the right) are done using the FDTD method, and using a perfectly matched layer (PML) boundary condition. Here the line source in air is aligned along the $y$ direction and lies $2 a$ above the interface, which is defined by the horizontal lines in Figs. 5(a)-5(d). 


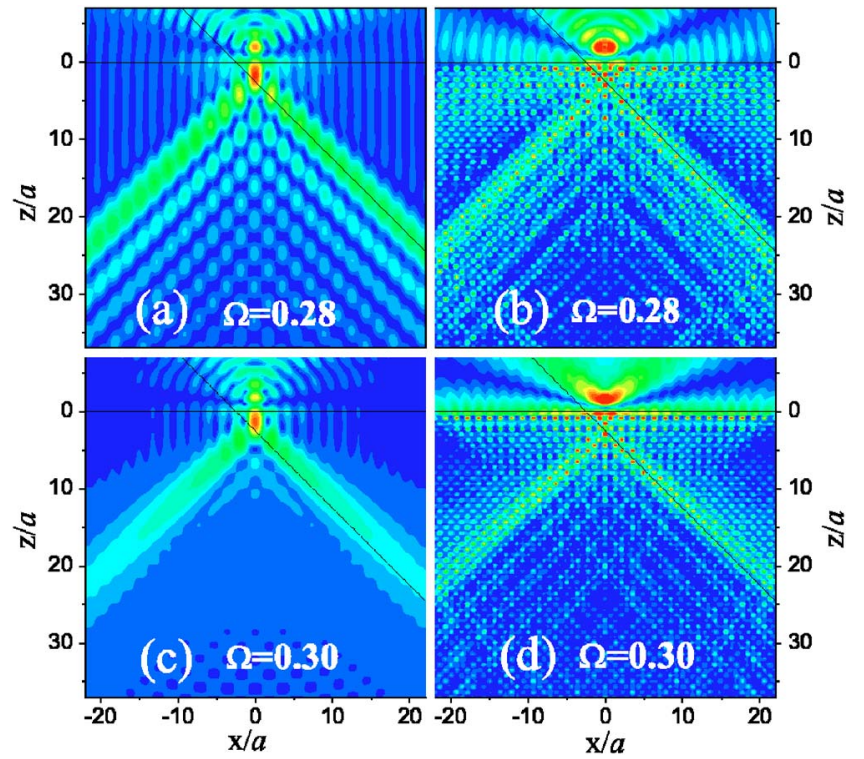

FIG. 5. (Color online) Light from a line source traveling through an air/PhC interface. On the left: amplitude of the $\mathbf{H}$ field calculated using the FIT at two different frequencies 0.28 (a) and 0.30 (c). On the right: corresponding fields calculated using the FDTD method. The horizontal lines indicate the interfaces, and the sloped lines are a guide for the eyes.

For $\Omega=0.28$, it can be seen from Fig. 5(a) that the PhC first focuses light to a point which then diverges into two branches subtending angles of $45^{\circ}$ with respect to each side of the interface, as expected from the square shape of the EFS. The field above the interface is in air, and it is an interference pattern between the incident and reflected fields. Figure 5(b) is the corresponding field pattern calculated using the FDTD method, and it agrees well with the results from the FIT. Comparing Fig. 5(b) with Fig. 5(a), we can see that the field inside the $\mathrm{PhC}$ has less fine structure when calculated using the FIT. The difference partly comes from the fact that the FIT is an effective medium approach which averages out the fine structure of the $\mathrm{PhC}$. The finite grid size used in the FDTD simulation also affects the agreement, as the FDTD method gives a better agreement with the FIT results after we reduced the grid size from $1 / 16 a$ to $1 / 32 a$. The bottom two figures are the field patterns for $\Omega=0.3$. Compared with the field patterns at $\Omega=0.28$ [Fig. 5(a)], the two branches at $\Omega=0.3$ [Fig. 5(c)] are better focused in the region near the interface and spread slowly further into the $\mathrm{PhC}$. This difference can be attributed to the fact that the EFS at $\Omega=0.3$ is more circular than the one at $\Omega=0.28$, which results in better focusing than collimation at $\Omega=0.3$. Again the result calculated using the FDTD, as shown in Fig. 5(d), agrees well with the FIT result in Fig. 5(c). The advantage of the FIT is that it is physically intuitive and it is much faster than the FDTD method. With the calculated EFS's, the typical simulation time of the FIT is less than 1 min even using an ordinary computer; while the FDTD method takes many hours using a much faster dedicated computer.

In practice, it is more interesting to study the imaging properties of a finite slab of $\mathrm{PhC}$ material. For simplicity, we start with a $\mathrm{PhC}$ slab in air; i.e., media (1) and (3) are the

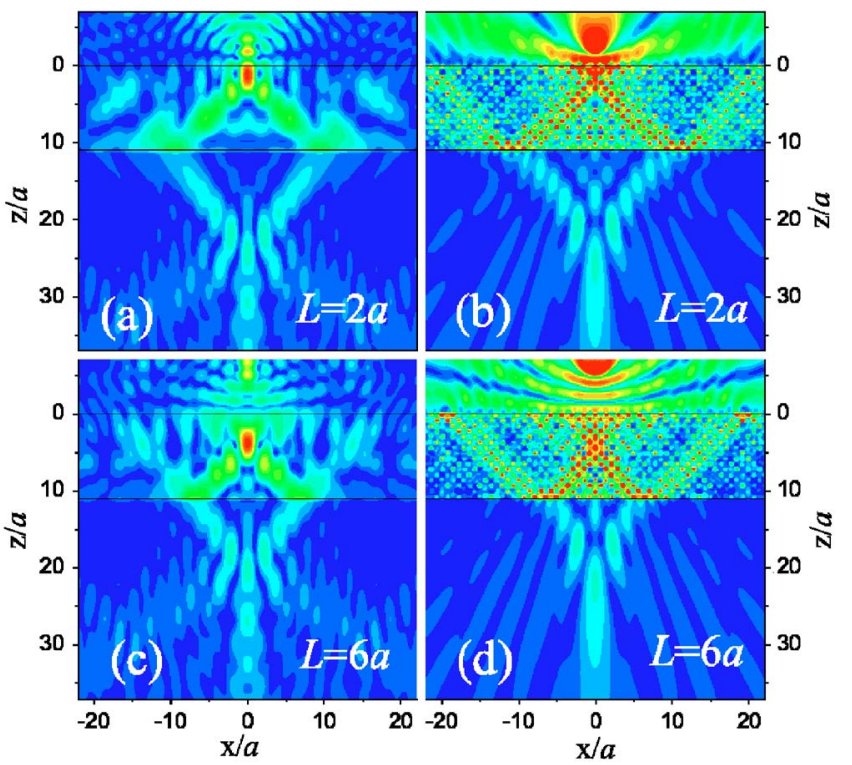

FIG. 6. (Color online) Field patterns at frequency 0.3 in and through a PhC slab with the source $2 a$ (top two figures) and $6 a$ (bottom two figures) away from the sample surface. Again figures on the left are calculated using the FIT and those on the right are done using the FDTD method. The horizontal lines define the top and bottom boundaries of the slab.

same. Figure 6 gives the field simulations at $\Omega=0.3$. Here the $\mathrm{PhC}$ slab is $8 \sqrt{2} a$ thick and the structure of the slab is shown in Fig. 1. Again field patterns on the left [Figs. 6(a) and 6(c)] are calculated with the FIT and those on the right [Figs. 6(b) and 6(d)] are from the FDTD method, where the boundary is chosen as shown in Fig. 1. We also found that different boundary conditions can affect light coupling as discussed in a recent paper. ${ }^{6}$ First we will discuss Figs. 6(a) and 6(b), which are the results when the source is $2 a$ away from the sample. The overall agreement between the FIT and FDTD methods is very good. Both the FIT and FDTD methods show that light is focused near the first interface in the $\mathrm{PhC}$ and there is a clear negative refraction happening at the second interface. To understand this better, we can take a look at the EFS in reciprocal space, as shown in Fig. 3. When light is coupled into the sample through the first interface, the shape of the EFS "causes" strong focusing of light (i.e., for small $z$ ) which then diverges and collimates. After the second interface, the EFS in air bends the light back and refocuses it. It can also be seen in Figs. 6(a) and 6(b) that there is a large reflection at both interfaces, due to the difference in the vertical component of the wave vectors in air $k^{a}$ and the PhC $k^{\prime}$ as shown in Fig. 3. Thus the coupling efficiency is fairly low, and this frequency range is not the most suitable for imaging. Comparing the field patterns calculated using the FIT and FDTD methods [e.g., Figs. 6(c) and 6(d), respectively], we can see that there are some surface modes along the interfaces in the FDTD simulation which are not included in the FIT. As can be seen, these surface modes lead to changes in the near-field image. By comparing with the full FDTD calculation, we found that the effect of surface modes is minor more than $\lambda$ (the wavelength in air) away from the crystal. However, the surface modes can be included in the FIT in a 


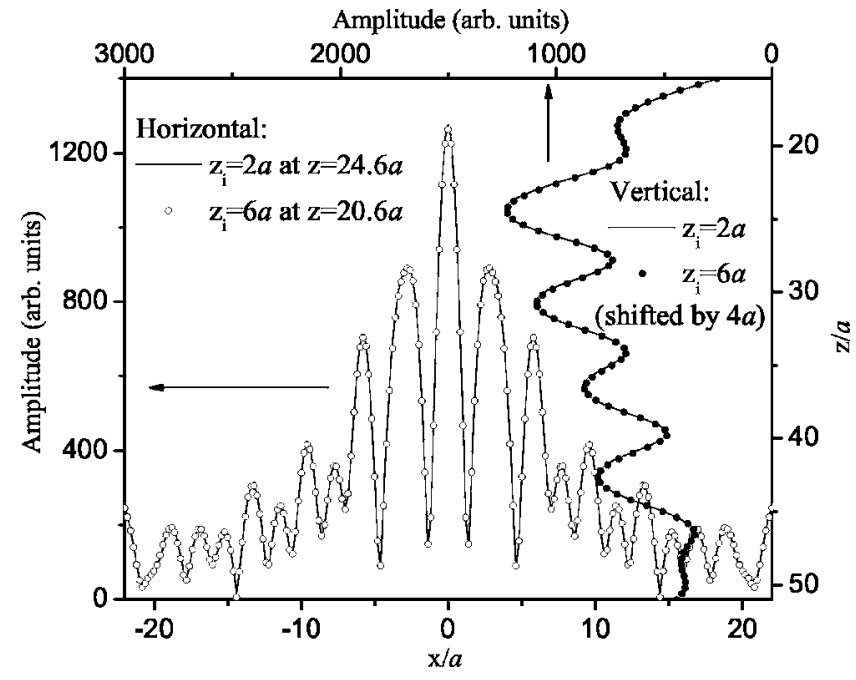

FIG. 7. Horizontal and vertical cross sections of the imaging field in Figs. 6(a) and 6(c). Here $z_{i}$ gives the distance between the source and the top sample surface. The horizontal cross sections are taken at $z=24.6 a$ (solid line) for $z_{i}=2 a$ and $z=20.6 a$ (circles) for $z_{i}=6 a$, respectively; the vertical cross section is taken along the center line $x=0$, and the one for $z_{i}=6 a$ (dots) has been shifted so that it can be compared directly with the one for $z_{i}=2 a$ (solid line).

situation where they are critical, such as subwavelength imaging as discussed in detail by Luo et al.,${ }^{13}$ where the source is $0.1 a_{s}$, roughly $0.03 \lambda$ away from the sample surface, and the center of the image is $0.16 \lambda$ away from the sample surface.

We can also determine the "lens formula" for our $\mathrm{PhC}$ slab by moving the source further away from the sample. Figures 6(c) and 6(d) give the results when the source is $6 a$ away from the sample surface. The agreement between the FIT and FDTD methods is again good. Both methods give the same result in that the images inside the $\mathrm{PhC}$ move further away from the sample surface. This property that the image distance increases or decreases as the source distance increases or decreases is similar to the imaging property of a mirror but with a real 2D image. Compared with Fig. 6(a), it also seems that in Fig. 6(c) the shape of the image field on the other side of the slab does not change but simply moves closer to the sample. To look at this in detail, we extract the horizontal and vertical cross sections of Figs. 6(a) and 6(c) and plot them in Fig. 7. Here the horizontal cross section of Fig. 6(a) (indicated with the solid line) is taken at a distance $z=24.6 a$ and the cross section of Fig. 6(b) (indicated with the circles) is performed at $z=20.6 a$. It can be seen that they overlap with each other exactly with the difference in distance $4 a$, which is the same as the shift of the source. The vertical cross section is taken along $x=0$, the solid line is for $z_{i}=2 a$, and the dots are for $z_{i}=6 a$ which has been shifted by $4 a$ for comparison, where $z_{i}$ is the distance between the source and the top surface of the sample. Again, the vertical cross sections overlap exactly. The good agreement for both vertical and horizontal cross sections shows that the imaging field pattern has only been shifted by $4 a$, the same amount as the change of the source distance. We also performed calculations with different source distances and concluded that the

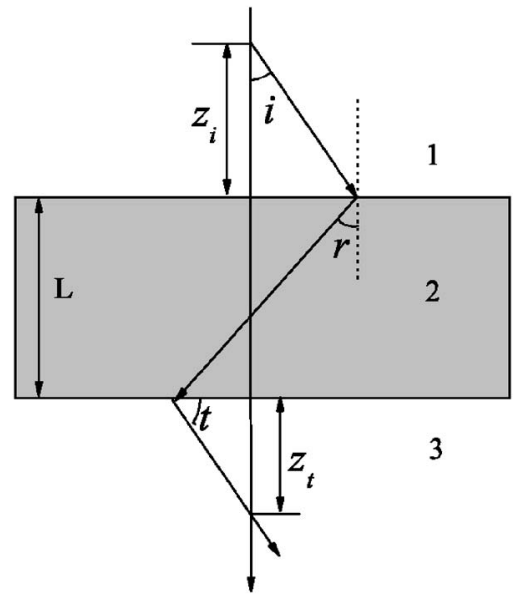

FIG. 8. Ray diagram of beam propagation, where $i$ is the incident angle in medium (1), $r$ the refracted angle in the $\mathrm{PhC}$, and $t$ the transmitted angle in medium (3), $L$ the thickness of the slab, $z_{i}$ and $z_{t}$ the source distance and image distance, respectively.

sum of the source distance and the image distance is a constant. The results from the FDTD method-i.e., Figs. 6(b) and 6(d) - show the same property. For simplicity, we will not plot their cross sections here. In fact, this relation is valid because media (1) and (3) are the same and it can be easily understood using the FIT method. For any stable light source, such as the monochromatic source that we consider there, the amplitude distribution of the wave vectors of incident waves at the sample surface do not change when we move the source further away from the PhC slab. From Eq. (2), we can see that the phase change caused by translating source $A$ can be compensated by translating the image along the $z$ axis by the same amount, because media (1) and (3) are the same (both are air) in the cases studied here.

When media (1) and (3) are different uniform materials with refractive index $n_{1}$ and $n_{3}$, respectively, we can derive the relation between the source distance $z_{i}$, image distance $z_{t}$, and the slab thickness $L$ with the help of the ray diagram of Fig. 8,

$$
z_{i} \tan (i)+z_{t} \tan (t)=L \tan (r)
$$

where $i$ is the angle of incidence in medium (1), $r$ the angle of refraction in the $\mathrm{PhC}$, and $t$ is the angle of transmission in medium (3), respectively. Equation (7) can be written more explicitly in terms of $i$,

$$
z_{i} \frac{\sin (i)}{\sqrt{1-\sin (i)^{2}}}+z_{t} \frac{n_{1} \sin (i)}{\sqrt{n_{3}^{2}-n_{1}^{2} \sin (i)^{2}}}=L \tan (r) .
$$

Here $r$ is a function of $i$, since $r$ is determined by the EFS of the PhC and depends on the incident direction $i$ at a certain frequency. For simplicity, we do not define a "refractive index" for the PhC, but keep $r$ as expressed in Eq. (8). It can be seen that Eq. 8 can be further reduced to a simple equation when media (1) and (3) are both air:

$$
z_{i}+z_{t}=L \tan (r) / \tan (i) .
$$


Since for a specific incident direction $i$ and $r$ are constant, Eq. (9) implies that $\Delta z_{i}+\Delta z_{t}=0$. In other words, the imaging field for any incident direction $i$ or any specific $k_{x}$ will change by the same amount $-\Delta z_{i}$ when we move the line source-i.e., changing the $z_{i}$, by $\Delta z_{i}$. Thus the whole image field will simply shift as the object is moved, as we have seen in Fig. 6.

In summary, the image inside or through a $\mathrm{PhC}$ slab is a real 2D (for a 2D slab) or 3D image (for a 3D PhC) and there is no central optical axis, which is different from the imaging properties of a traditional lens as Notomi has pointed out. ${ }^{14}$ The reason is that the phase change in a lens which is responsible for focusing is only determined by the shape of the lens which is usually symmetric and has a central axis. However, in a $\mathrm{PhC}$ slab the phase change is controlled by the shape of EFS's, and the imaging is not due to a spatial variation of the optical path length as in a conventional lens, but is instead due to the variation of the "effective index" with the propagating direction inside the $\mathrm{PhC}$. Another distinct imaging property of $\mathrm{PhC}$ is that the sum of the source distance and the image distance is a constant when the media on both sides of the slab are the same. Also a PhC slab does not focus a plane-wave input. In other words, if a pure plane wave propagates through the crystal, it remains a plane wave and is not focused, which is distinct from a conventional lens.

\section{CONCLUSION}

In this paper, the FIT method was used to study light propagation in and through $\mathrm{PhC}$ structures using an effective medium approximation based on EFS calculations. In particular, bending, splitting, and focusing phenomena were studied in a 2D PhC slab. Light emitted from a line source outside of the slab can be focused and divided within the $\mathrm{PhC}$; subsequently, the light may be bent negatively and refocused again on the other side of the slab. The imaging properties of the $\mathrm{PhC}$ are discussed in comparison to a conventional lens. The results calculated using the FIT method agree well with the field calculated using a FDTD method. Compared with the FDTD method, the FIT approach is more physically intuitive and significantly faster to implement. This effective medium technique can also be readily modified and applied to more complicated $\mathrm{PhC}$ structures and/or heterostructures.

\section{ACKNOWLEDGMENTS}

We wish to acknowledge the support of NSERC, CIPI, AFOSR, and CSA and to thank Dr. Selvakumar Nair for helpful discussions and providing the Unix platform which was used for the dispersion relation simulation.
*Electronic address: harry.ruda@utoronto.ca

${ }^{1}$ E. Yablonovitch, Phys. Rev. Lett. 58, 2059 (1987).

${ }^{2}$ S. John, Phys. Rev. Lett. 58, 2486 (1987).

${ }^{3}$ P. V. Parimi, W. T. Lu, P. Vodo, J. Sokoloff, J. S. Derov, and S. Sridhar, Phys. Rev. Lett. 92, 127401 (2004).

${ }^{4}$ A. Berrier, M. Mulot, M. Swillo, M. Qiu, L. Thylén, A. Talneau, and S. Anand, Phys. Rev. Lett. 93, 073902 (2004).

${ }^{5}$ S. He, Z. Ruan, L. Chen, and J. Shen, Phys. Rev. B 70, 115113 (2004).

${ }^{6}$ S. Xiao, M. Qiu, Z. Ruan, and S. He, Appl. Phys. Lett. 85, 4269 (2004).

${ }^{7}$ K. Guven, K. Aydin, K. B. Alici, C. M. Soukoulis, and E. Ozbay, Phys. Rev. B 70, 205125 (2004).

${ }^{8}$ A. Martinez, H. Miguez, A. Griol, and J. Marti, Phys. Rev. B 69, 165119 (2004).

${ }^{9}$ P. V. Parimi, W. T. Lu, P. Vodo, and S. Sridhar, Nature (London) 426, 404 (2003).

${ }^{10}$ S. Foteinopoulou, E. N. Economou, and C. M. Soukoulis, Phys. Rev. Lett. 90, 107402 (2003).

${ }^{11}$ S. Foteinopoulou and C. M. Soukoulis, Phys. Rev. B 67, 235107 (2003).

${ }^{12}$ E. Cubukcu, K. Aydin, E. Ozbay, S. Foteinopoulou, and C. M. Soukoulis, Phys. Rev. Lett. 91, 207401 (2003).
${ }^{13}$ C. Luo, S. G. Johnson, J. D. Joannopoulos, and J. B. Pendry, Phys. Rev. B 68, 045115 (2003).

${ }^{14}$ M. Notomi, Opt. Quantum Electron. 34, 133 (2002).

${ }^{15}$ C. Luo, S. G. Johnson, J. D. Joannopoulos, and J. B. Pendry, Phys. Rev. B 65, 201104(R) (2002).

${ }^{16}$ V. G. Veselago, Usp. Fiz. Nauk 92, 517 (1964).

${ }^{17}$ C. H. Kuo and Z. Ye, Phys. Rev. E 70, 026608 (2004).

${ }^{18}$ E. Istrate and E. H. Sargent, IEEE J. Quantum Electron. 41, 461 (2005).

${ }^{19}$ S. G. Johnson and J. D. Joannopoulos, Opt. Express 8, 173 (2001).

${ }^{20}$ S. Yang, J. H. Page, Z. Liu, M. L. Cowan, C. T. Chan, and P. Sheng, Phys. Rev. Lett. 93, 024301 (2004).

${ }^{21}$ X. Yu and S. Fan, Appl. Phys. Lett. 83, 3251 (2003).

${ }^{22}$ J. H. Page, A. L. Goertzen, S. Yang, Z. Liu, C. T. Chan, and P. Sheng, Photonic Crystals and Light Localization in the 21st Century, edited by C. M. Soukoulis (Kluwer Academic, Amsterdam, 2001), pp. 59-68.

${ }^{23}$ J. H. Page, S. Yang, M. L. Cowan, Z. Liu, C. T. Chan, and P. Sheng, Wave Scattering in Complex Media: From Theory to Applications, edited by B. A. van Tiggelen and Sergey Skipetorv (Kluwer Academic, Amsterdam, 2003), pp. 283-307. 